\title{
Oportunidades del Acuerdo de Cooperación Ambiental de América del Norte en la era post-TLCAN
}

\author{
Felipe Adrián Vázquez Gálvez, Liliana Rivera-Lozano
}

\begin{abstract}
Resumen: El ACAAN actúa como una plataforma independiente de cooperación internacional entre Estados Unidos, México y Canadá para el desarrollo de protocolos de protección del medio ambiente. La CCA se concibe como una práctica herramienta para la instrumentación de las metas trazadas por el ACAAN. En consecuencia, la CCA se encarga de gestionar, supervisar e implementar diversas estrategias de conservación, protección, y mejoramiento del medio ambiente por medio de la colaboración entre la sociedad civil y el sector gubernamental. En la actualidad, el debate en torno a si la reciente revisión y ratificación del TLCAN requerirá mantener el existente funcionamiento del ACAAN permite el planteamiento de nuevas áreas de oportunidad en vista del actual contexto político, económico y ambiental global.
\end{abstract}

Palabras clave: Canadá, comercio, cooperación ambiental, Estados Unidos, trinacional, México.

El presente artículo es un extracto de la conferencia magistral dictada por el autor principal en el Centro de Investigación y Docencia Económica (CIDE), el 31 de octubre 2017 en Aguascalientes, México. La conferencia fue parte de los trabajos del Congreso Internacional del Consorcio para la Investigación Comparada sobre Integración Regional y Cohesión Social (RISC). La serie de conferencias organizadas por el CIDE, el Instituto de Ecología (INECOL), la Universidad de Helsinki y la Universidad de Luxemburgo logró atraer la atención y participación de investigadores de diferentes partes del mundo. Posterior a la presentación, surgió un interesante debate acerca de la nueva perspectiva que ofrece el Tratado de Libre Comercio de América del Norte (TLCAN) y el paralelo Acuerdo de Cooperación Ambiental de América del Norte (ACAAN) en vista de la 
postura ambiental adoptada por la actual administración del Presidente Trump, así como las posibles implicaciones que traerán consigo su inminente revisión.

En este artículo los autores nos preguntamos si después de más de 20 años de implementación de las medidas de apertura comercial relacionadas con el TLCAN, servirá examinar y reacondicionar el ACAAN o bien, se procederá a derogarlo y asimilarlo como parte del TLCAN. El ACAAN ha resultado particularmente exitoso en atender los asuntos ambientales más apremiantes de la región por medio de la fundación de la Comisión de Cooperación Ambiental de América del Norte (CCA). Logrando mantener la región en una posición de liderazgo global en el desarrollo de agendas como el adecuado manejo de sustancias químicas toxicas y el derecho público a conocer sus riesgos ambientales asociados. Motivo por el cual, es necesario analizar la posibilidad de mantener la vigencia del ACAAN y enfatizar su particular relevancia dentro del actual esquema de colaboración internacional en materia medio ambiental subsecuente a la firma del TLCAN en 1994.

\section{Los tratados de libre comercio y el medio ambiente en un marco histórico}

Durante la época posterior a la segunda guerra mundial, los países involucrados en el conflicto empezaron a trabajar en pro de enmendar los quebrantados lazos de confianza internacionales y adquirir una razonable estabilidad económica para sus habitantes. Dichas aspiraciones se vieron materializadas mediante la formación del Acuerdo General sobre Aranceles Aduaneros y Comercio conocido como GATT por sus siglas en inglés, firmado en noviembre 1947 (Gallagher, 2004). Bajo la supervisión del GATT, las 23 naciones participantes consiguieron reactivar y fortalecer sus mercados internacionales, así como fomentar un estado de paz global.

A principios de los años 90, el nuevo panorama global presentaba la oportunidad de transformar la GATT en una organización que respondiera a la formación de nuevas políticas comerciales enfocadas a conseguir un crecimiento constante y eficiente de la economía internacional. A partir del primero de enero de 1994, GATT se conocería como la Organización Mundial de Comercio (WTO) (Vaughan, 2017). Durante tal periodo de grandes cambios comerciales fue que se diseñó el TLCAN. El cual se conformó como el primer acuerdo comercial formado por naciones con diferentes niveles de desarrollo socio-económico: Canadá, los Estados Unidos Mexicanos y los Estados Unidos de América, lo cual ya por sí mismo representaba un parte aguas para los tratados hasta entonces en funcionamiento. 
El tratado comercial buscaba conseguir una mayor apertura económica entre los países participantes y con esto aumentar la accesibilidad entre mercados comerciales internacionales que permitiera mayor movilidad de capital y recursos humanos que fomentara más posibilidades de desarrollo y progreso para los ciudadanos. En adición a ello, el TLCAN requirió la negociación de una conveniente agenda medio ambiental en respuesta al debate climatológico y de protección de especies presentado por organizaciones de Origen No Gubernamental (ONGs) y Organizaciones de la Sociedad Civil (OSCs) en su mayoría pertenecientes a los Estados Unidos. Estas organizaciones, solicitaron la creación de un organismo independiente a los intereses políticos y económicos de los gobiernos de cada país, capaz de asegurar el correcto funcionamiento y apego a las regulaciones establecidas en el tratado. En agosto de 1993, se pactó la formación del acuerdo paralelo a TLCAN, el ACAAN, el cual ayudo a concluir las negociaciones del TLCAN con el apoyo de la sociedad norteamericana (Johnson \& Beaulieu, 1996).

\section{Organismos de protección ambiental bajo la interpretación del TLCAN}

A pesar de que las negociaciones entre las tres naciones para conformar el TLCAN y su acuerdo paralelo el ACAAN duraron varios años, estos no entraron el vigor sino hasta el primero de enero de 1994. Los anexos de protección del medio ambiente establecidos en el esqueleto del TLCAN se enfocaron en perseguir la homologación de estándares y regulaciones entre los Estados Unidos, México y Canadá con el fin de disminuir el impacto ambiental causado por las actividades propias a la implementación misma del tratado. Análogamente, el ACAAN se dio a la tarea de edificar una nueva organización encargada de instrumentar la cooperación y participación ciudadana en contexto de los apéndices ambientales incorporados al TLCAN. De esta forma, se consolido la CCA entre los países de la región de América del Norte (Hufbauer, Esty, Orejas, Rubio, \& Schott, 2000).

De acuerdo a la plataforma virtual del Comisión de Cooperación Ambiental administrada por el Secretariado (2018), la visión general de la organización se establece bajo el lema: “Tres países unidos para la protección del medio ambiente que compartimos" el cual busca resonar en la conciencia socio-ambiental de las tres naciones. Su slogan resulta claro, tanto la contaminación como los fenómenos naturales ocasionados por el cambio climático son indiferentes a las divisiones y fronteras geopolíticas de las naciones, por lo que necesitamos trabajar en conjunto para corre- 
gir y reparar los daños ambientales de la región. La misión de la CCA es gestionar y arbitrar la unión de esfuerzos intergubernamentales y de la sociedad civil con el apoyo de científicos e investigadores independientes a favor de la protección, conservación y mejoramiento del medio ambiente en América del Norte. (CEC Secretariat, 2018).

La manera en la que la CCA consigue involucrar tanto al gobierno como a la ciudadanía de los tres países en un solo proyecto es por medio de la formación de tres estructuras separadas con actividades únicas: el consejo general, el comité consultivo público también conocido como J-Pack y el secretariado tal y como se puede observar en el esquema de la figura 1.

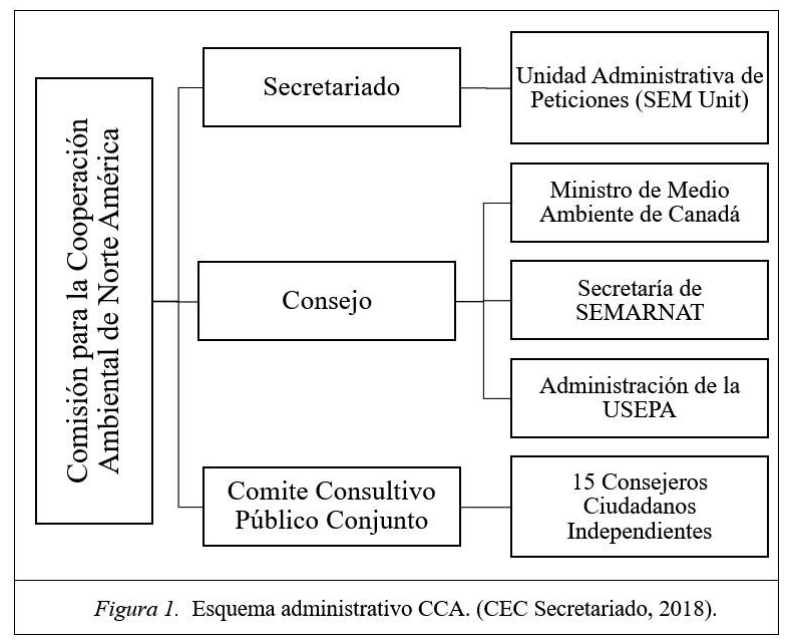

El consejo general es el órgano superior de la CCA formado por el ministro o secretario ambiental de cada país los cuales se desempeñan como un enlace directo para el diálogo y la discusión entre las distintas partes del CCA y los gobiernos trinacionales. Por su parte el comité consultivo, se compone de 15 ciudadanos voluntarios, cinco por cada país participante, los cuales se encargan de orientar y vigilar el inequívoco cumplimiento de las regulaciones ambientales establecidas. También se encarga de estudiar la factibilidad y proponer un presupuesto financiero para estudios científicos independientes del medio ambiente. Finalmente, el secretariado provee apoyo administrativo, técnico y operativo a la comisión, cumple con la gestión de la agenda de trabajo y dirige la unidad de peticiones sobre la aplicación efectiva de la legislación ambiental también conocida como Unidad SEM (CEC Secretariat, 2018).

La Comisión de Cooperación Ambiental, constantemente realiza funciones concretas a favor del medio ambiente tales como el análisis del 
impacto ambiental resultante del ejercicio derivado del TLCAN. Diseña métodos de prevención, control y remediación de la contaminación generada por las actuales prácticas comerciales internacionales. Promueve la integración de industrias energéticas sustentables o con un menor impacto ambiental y busca formar estrategias de adaptación al cambio climático. En adición, lucha por la protección y el manejo adecuado de especies nativas para asegurar la conservación de la biodiversidad de la región y contempla la integración de comunidades rezagadas a servicios de salubridad básicos (Johnson \& Beaulieu, 1996).

Así mismo, la CCA se encarga de promover el intercambio de información de forma segura y transparente entre instituciones privadas y gobierno por parte de los tres países participantes. Tomemos como ejemplo, la realización de un estudio de riesgo de salud por partículas suspendidas de un área urbana concurrida; los investigadores independientes, instituciones de seguridad y centros de salud, al igual que los habitantes de la zona tienen derecho a acceder libremente a los resultados generados por el estudio. El derecho a compartir abiertamente información entre gobiernos y particulares es sin lugar a dudas uno de los principales debates en la actualidad a nivel global (Vaughan, 2017). Esta discusión enfocada al ámbito medio ambiental promete conducirnos a un estado de empoderamiento social que fomente el fortalecimiento de la justicia ambiental. Podemos resumir que la función esencial de la CCA es certificar el desarrollo e implementación de regulaciones ambientales adecuadas y homogéneas para la región Norte del continente Americano.

\section{Una visión a futuro de la cooperación medio ambiental para un desarrollo sustentable}

Después de más de veinte años de promulgado el ACAAN la pregunta obligada es si la CCA podría servir a los retos emergentes en el tema de comercio y política ambiental. Esta reflexión pasa sin duda por el análisis de las lecciones aprendidas y de los avances que se dieron durante la primera etapa de implementación del TLCAN. Nos focalizamos en particular en la agenda de cooperación que se desarrolló a partir de planes quinquenales en los que las partes acordaron acciones específicas para atender retos en el estudio o la evaluación de problemáticas que no se podían entender fuera del contexto regional. Tales como el uso de plaguicidas, de contaminantes tóxicos persistentes, la contribución de las emisiones del sector de generación de electricidad y la relevancia de estudiar las especies prioritarias de la región en el contexto de sus espacios, por mencionar algunas. 
Dentro de las lecciones aprendidas estos últimos 20 años, se reconoce que la plataforma de colaboración de la CCA ha sido particularmente exitosa en atender los asuntos ambientales más apremiantes en la escala subcontinental. Entre otras cosas ha permitido que la región se mantenga en una posición de liderazgo en la implementación de agendas globales en el manejo de sustancias químicas tóxicas y en el derecho a conocer los riesgos ambientales asociados a estas. En cuanto a la amenazas de un posible "paraíso para contaminantes" discutido por múltiples economistas ambientales (Gallagher, 2004), que condicionó en parte la arquitectura del ACAAN y su contra parte laboral TLCAN es una discusión que ha se ha disipado en respuesta a las actuales condiciones generales del medio ambiente en Norte América en vista de la relativa mejoría en las condiciones salariales sobre todo en la región fronteriza de México y los Estados Unidos (Aguilera \& Castro, 2018) generadas en gran partepor la creación de instituciones mexicanas tales como la SEMARNAT, la PROFEPA y muy recientemente junto con la reforma energética de México la Agencia de Seguridad Energía y Ambiente (ASEA). Organismos quienes cuentan con la suficiente fortaleza institucional como para ofrecer las condiciones mínimas de protección al medio ambiente. De ahí que el enfatizar en una negociación comercial el posible daño ambiental ha dejado de ser una excusa. El nuevo reto se presenta en convertir la protección del medio ambiente a un nivel global y continental de una excusa a una genuina preocupación y ocupación de la cooperación ambiental trinacional.

El 16 de agosto 2017, se iniciaron pláticas para la actualización y una posible renegociación del TLCAN, las cuales se han centrado en tratar los aspectos comerciales. Con respecto a las iniciales preocupaciones ambientales manifestadas durante las primeras fases de actividad del TLCAN, empezaron a disiparse lentamente en medida que surgieron diferentes problemáticas migratorias y laborales resultantes del establecimiento de un mayor número de lazos comerciales entre las naciones participantes. Al mismo tiempo, que diversas problemáticas ambientales dentro del orden regional sufrieron de una paulatina disminución de atención y soporte brindado por parte de organizaciones de tipo ONGs y OSCs quienes priorizaron sus esfuerzos al terreno global, afectando así drásticamente la capacidad de acción de organizaciones subcontinentales como la CCA (Johnson, Page, Haverkamp, Mizroch, Basurto, \& Torres, 2004; CEC Secretariat, 2016). Los beneficios directos del TLCAN se han enfocado en el bienestar de grandes corporaciones quienes han construido una robusta cadena de integración de sus productos, eficiente comercialmente y con esquemas relativamente homologados de calidad ambiental. Sin embargo, en lo referente a las actividades para la cohesión social y monitoreo ambiental comunitario, así como en el desempeño de las autoridades en 
este rubro, identificamos un fenómeno de inflexión que en algunos casos se ha llegado a percibir como un retraimiento de la actividad de las OSC ambientales.

Esto es a su vez resultado del preponderante interés de la sociedad civil con respecto al tema de la violencia, la migración y los derechos humanos junto con la agobiante preocupación de actividades terroristas en la era posterior al ataque de las torres gemelas el 11 de septiembre 2001. Los cuales han entorpecido y ralentizado la movilidad de bienes y personas a través de las fronteras internacionales a pesar de mantenerse en efecto los tratados comerciales como el TLCAN, engrosando así la división geográfica entre los tres países.

A partir de estas lecciones aprendidas en las pasadas décadas, podemos plantear cuatro ejes importantes de una posible agenda post-TLCAN en el marco del actual del ACAAN. El primero, sería el fomentar el desarrollo de una matriz energética continental que de manera inteligente se oriente al desacoplamiento del crecimiento económico y la actividad comercial del consumo de carbono. En segundo término, continuar con el saneamiento básico de las comunidades más apartadas con énfasis en el mejoramiento de la salud humana. Como tercer punto, la imperante necesidad de atender el constante mejoramiento de los canales de comunicación de riesgo bajo un esquema de empoderamiento de la sociedad y de la justicia ambiental. Finalmente, reforzar el fortalecimiento de la conservación de especies y espacios relevantes para especies de vida silvestre prioritarias.

De manera trasversal a estos ejes planteamos cuatro líneas de trabajo. Siendo la primera la combinación de estrategias para reducir la huella de carbono mediante la concientización de la comunidad y las instituciones reguladoras. Promover esquemas de ciudades resilientes como una maniobra de mejoramiento de la calidad de vida y de adaptación al cambio climático. Finalmente, explorar esquemas innovadores de asociación entre el sector privado, gobierno y sociedad. Con la intención compartida de homologar estándares y reglamentos sobre todo aquellos encaminados a medir y monitorear la calidad ambiental en las cuencas hidrológicas, litorales y atmosféricas.

\section{Conclusión}

La apertura comercial entre los Estados Unidos, México y Canadá resultante de la implementación del TLCAN trajo consigo la irreversible distorsión de la actividad económica, social y cultural de estas naciones. Un ejemplo claro se percibe en materia ambiental, donde el ACAAN impulso 
la modernización del marco institucional encargado de protección conservación y mejoramiento del medio ambiente, en especial para México. Hoy en día, México se plantea a sí mismo como un entusiasta participante en la arena internacional en temas de relevancia global tales como el cumplimiento de los 17 retos de sustentabilidad establecidos por la Organización de las Naciones Unidas. En general, la región norte del continente americano ha trabajado íntimamente en colaboración acrecentar sus capacidades de la reacción a iniciativas globales en materia de manejo de sustancias tóxicas, conservación de especies emblemáticas amenazadas, el combate y mitigación del cambio climático. Buscando plantear nuevas metas para un futuro próximo, tales como diseñar e implementar estrategias de remediación y adaptación al cambio climático para el desarrollo sustentable de nuestra sociedad y supervisar la construcción de sistemas de generación de energía alternativas entre otros.

Durante los últimos meses y en vista de la postura adoptada por la actual administración de los Estados Unidos; México y Canadá han intentado formar una alianza para ejercer presión sobre su compañero comercial que promueva la positiva ratificación de protocolos ambientales vinculantes a nivel regional y global. En reacción a esto, la administración del presidente Trump ha tomado una actitud desafiante y agresiva amenazando con dar por terminados tratados de índole comercial, en particular el TLCAN, al cual a través del ACAAN vincula sus políticas ambientales de manera paralela. Meses antes se presentó la reconfiguración reglamentaria propuesta por los Estados Unidos al TLCAN la cual impactará de forma directa al ACAAN y con ello al presupuesto de fondo público destinado para la CCA. Siendo específicos, la iniciativa de la administración del presidente Trump propone adoptar una reducción al presupuesto anual de la CCA de dos millones de dólares (Vaughan, 2017).

Posteriormente, tomando ventaja de la inestable situación política de México con respecto al inminente y aparente profundo cambio de gobierno que llevara a cabo bajo el mando del nuevo presidente electo Andrés Manuel López Obrador, el equipo de trabajo del presidente Trump urgió la pronta firma de un preliminar acuerdo bilateral del TLCAN con el aún vigente gobierno mexicano. Dicho acuerdo preliminar fue firmado por el presidente Enrique Peña Nieto el pasado 27 de agosto 2018 y en él Mexico cedió ante las peticiones del mandatario estadounidense particularmente en referencia a la industria automotriz (Swanson, 2018). Hasta el día de hoy, se espera que para la primera semana del mes de septiembre del presente año el gobierno canadiense logre adherirse al preliminar acuerdo comercial que hasta este punto se presenta como un arreglo binacional entre Mexico y Estados Unidos. Por su parte el gobierno mexicano, anuncio que se espera conseguir la firma final del TLCAN 2.0 entre las tres 
naciones para el 30 de Noviembre del mismo año. Las consecuencias de no conseguir actualizar y mantener en funcionamiento el TLCAN junto con el ACAAN afectaran no únicamente a las relaciones internacionales y el ámbito económico-comercial de los países participantes, si no que alteraran significativamente el progreso de la actual agenda de justica ambiental y protección de biodiversidad en la región.

En vista de la complicada postura ambiental adoptada por los Estados Unidos, resulta clara la necesidad de mantener vigente y asegurar la autonomía del ACAAN dentro del presente contexto de renegociación del TLCAN 2.0. Si dentro de los siguientes meses los países de América del Norte no conseguimos asegurar la presencia, eficacia e imparcialidad de organizaciones tales como la CCA para el adecuado desarrollo e implementación de políticas de protección ambiental, las consecuencias sobre nuestra agenda de protección ambiental generaran severas repercusiones ambientales no solo en la región si no a escala global.

FELIPE ADRIÁN VÁZQUEZ GÁLVEZ realizo su licenciatura y maestría en Química en la Universidad de Texas en El Paso (UTEP). Recibió el grado de Doctor en Filosofía en el área de aerosoles atmosféricos del Instituto Mexicano de Tecnología del Agua. Se ha desempeñado como Fiscal Federal Auxiliar en PROFEPA, Subsecretario de Administración en SEMARNAT, Director Ejecutivo de la Comisión para la Cooperación Ambiental de América del Norte - CCA y Coordinador General del Servicio Meteorológico Nacional de México. A partir de 2014, trabaja en el Departamento de Ingeniería Civil y Ambiental de la Universidad Autónoma de Ciudad Juárez (UACJ). Email: fvazquez@uacj.mx. ORCID: 0000-0003-0282-8023

LILIANA RIVERA LOZANO obtuvo una licenciatura en Ingeniería Física de la Universidad Autónoma de Ciudad Juárez (UACJ) en 2015. Posteriormente, la Escuela de Ingeniería y Ciencia de Materiales (SEMS) de la Universidad de Londres Queen Mary (QMUL) el grado de Maestro de Ciencias en Ingeniería de Energías Sustentables en 2017. Actualmente es la jefa académica y de investigación del Laboratorio de Climatología y Calidad del Aire de UACJ. Email: riloliliana@gmail.com. ORCID: 0000-0001-8846-5643

\section{REFERENCIAS}

Aguilera, A., \& Castro, D. (2018). NAFTA and wage inequality in Mexico: An analysis for border cities, 1992-2016. Tijuana: Frontera Norte, 30(6), pp. 85-110.

CEC Secretariat (2016). 20 Years of NAFTA and the NAAEC. Summary of contributions in response to JPAC's call for information and comments. CEC, Montreal. Descargado de http://www.cec.org/sites/default/files/documents/summaryof-comments-oct16.pdf 
CEC Secretariat. (10 agosto 2018). North American Commission for Environmental Cooperation, Three Countries Working Together to Protect Our Shared Environment. Montreal. Descargado de http://www.cec.org

Gallagher, K. P. (2004). Free trade and the environment, Mexico, NAFTA, and beyond. California: Stanford University Press.

Hufbauer, G. C., Esty, D. C., Orejas, D., Rubio, L., \& Schott, J. J., (2000). NAFTA and the environment, seven years later. Washington, DC: Kirby Lithographic.

Johnson, P.M., \& Beaulieu, A. (1996). The environment and NAFTA, understanding and implementing the new continental law. Ontario: Island Press.

Johnson, P. M., Page, R., Haverkamp, J. A., Mizroch, J. F., Basurto, D., \& Torres, B. (2004). Ten years of North American environmental cooperation. Report of the Tenyear Review and Assessment Committee to the Council of the Commission for Environmental Cooperation. Montreal: CEC.

Swanson, A. (27 agosto 2018). México y Estados Unidos Alcanzan Acuerdo Preliminar para el TLCAN, Sin Canadá. The New York Times. Descargado de: https:// www.nytimes.com/es/2018/08/27/tlcan-mexico-estados-unidos-preliminar/

Vaughan, S. (junio 2017). Environmental record: History, outcomes, impacts and options. International Institute for Sustainable Development, Comentario (pp. 1-12). Winnipeg.

\title{
Opportunities of the North American Environmental Cooperation Agreement during the post-NAFTA era
}

Felipe Adrián Vázquez Gálvez, Liliana Rivera-Lozano

\begin{abstract}
NAAEC acts as an independent platform for the international cooperation between the United States, Mexico and Canada over a wide range of environmental protocols. The CEC was formed as a practical and valuable tool to achieve NAAEC goals. Therefore, the CEC supervises the development of environmental policies and their accurate implementation among the three nations. In addition, the CEC promotes the participation of private citizens in collaboration with their governments to develop diverse strategies for conservation, protection, and improvement of our shared environment. Whether or not it is necessary to maintain regular operations of NAEEC is a subject currently evaluated during the present renegotiations of NAFTA, which provides new opportunities in view of the contemporary political, economic and environmental scene.
\end{abstract}

Keywords: Canada, Commission for Environmental Cooperation (CEC), environmental policies, Mexico, North America Agreement on Environmental Cooperation (NAAEC), North American Free Trade Agreement (NAFTA), United States of America 
Opportunités de l'Accord nord-américain de coopération environnementale dans l'ère post-ALENA

\section{Felipe Adrián Vázquez Gálvez, Liliana Rivera-Lozano}

Résumé : L'ANACDE sert de plate-forme indépendante pour la coopération internationale entre les États-Unis, le Mexique et le Canada sur un large éventail de protocoles environnementaux. La CCE a été créée comme outil pratique et utile pour atteindre les objectifs de l'ANACDE. Par conséquent, la CCE supervise l'élaboration des politiques environnementales et leur mise en œuvre précise parmi les trois pays. En outre, la CCE encourage la participation des citoyens à l'élaboration de stratégies diverses en faveur de la conservation, la protection et l'amélioration de notre environnement commun. La question de savoir s'il est nécessaire ou non de maintenir les opérations régulières de l'ANACDE est actuellement débattue lors des renégociations de l'ALENA, qui ouvre de nouvelles perspectives sur les scènes politique, économique et environnementale contemporaines.

Mots-clés: Accord nord-américain de coopération environnementale (ANACDE), Accord de libre-échange nord-américain (ALENA), Canada, Commission de coopération environnementale (CCE), États-Unis d`Amérique, Mexique, politiques environnementales 